\title{
CARACTERIZACIÓN DE LAS HABILIDADES INVESTIGATIVAS EN LA PRODUCCIÓN DE TRABAJOS ACADÉMICOS
}

\author{
William Jesús Rojas Gutiérrez* \\ Universidad Católica Sede Sapientiae \\ williamtor@hotmail.com \\ Abel Alejandro Tasayco Jala* \\ Universidad Católica Sede Sapientiae \\ drabelalejandrotasaycojala@gmail.com
}

Fecha de recepción: agosto de $2020 \quad$ Fecha de aceptación: diciembre de 2020

Resumen: En el contexto integral del hombre, actualmente, se requiere evidenciar un conjunto de competencias generales y específicas, las mismas

\begin{abstract}
William Jesús Rojas Gutiérrez es maestro en Gestión Estratégica Empresarial. Licenciado en Educación, en la especialidad de Filosofía y Religión. Con estudios Filosóficos y Teológicos. Estudios de especialización en investigación: Metodología y Elaboración de Tesis de Posgrado, Formación en Redacción Académica Estilo APA (7.a Edición) y gestor de información Mendeley. Diplomado en Metodología de la Investigación en la Educación y Diplomado en Metodología de la Investigación Cualitativa. Diplomado en Métodos y técnicas de la Investigación. Diplomado en Currículo por Competencias y evaluación en Educación Superior. Experiencia en coordinación de Tutoría y Orientación Escolar (TOE). Tutoría universitaria. Capacitador de Turnitin y Urkund. Asesor y jurado de tesis. Docente de Investigación de Pre y Posgrado (Universidad Católica Sedes Sapientiae.) Docente de Investigación de Posgrado (Universidad Católica de Trujillo) - UCT, Docente de pregrado de la Universidad Privada San Juan Bautista - UPSJB y Universidad Tecnológica del Perú-UTP. Ha dado ponencias Internacionales en Bolivia, Chile, México y Ecuador.
\end{abstract}


que permiten una preparación integral de los futuros profesionales para enfrentar las expectativas del dinámico mercado laboral. En este sentido, es necesario el desarrollo de competencias investigativas en los estudiantes universitarios a fin de producir científicamente. Por ello, la presente investigación pretendió caracterizar las habilidades investigativas que debe presentar los estudiantes de educación superior para lograr plasmar constructos científicos. La metodología utilizada fue la revisión sistemática de la literatura científica con el propósito de determinar el estado de la cuestión, siendo la discusión a través de fuentes primarias que se aproximen al tema de estudio, los mismos que contribuirán al análisis crítico y riguroso. Para lograrlo, se emplearon buscadores académicos de revistas científicas de alto impacto de los últimos cinco años. En síntesis, a través del nivel sistémico se determinaron las particularidades de las habilidades investigativas respecto de la producción científica exitosa de los estudiantes universitarios.

Palabras Clave: Aptitud, Actividad intelectual, competencias para la vida, competencias profesionales, investigación transdisciplinaria, programa de investigación.

\section{CHARACTERIZATION OF RESEARCH SKILLS IN THE}

** Abel Alejandro Tasayco Jala es doctor en Psicología Educacional y Tutorial por la Universidad Nacional de Educación, doctorando en Administración (Universidad Cesar Vallejo), magíster en Docencia Universitaria (Universidad Nacional de Educación), licenciado en Administración y licenciado en Educación Primaria (Universidad Nacional San Luis Gonzaga de Ica). Docente en Pregrado (Universidad Católica Sedes Sapientiae, Universidad Privada San Juan Bautista, Universidad Autónoma de Ica). Docente de Posgrado (Convenio Interinstitucional Universidad Nacional San Luis Gonzaga de Ica - Universidad Autónoma de Ica). Excoordinador del Área de Investigación y de Psicopedagoga del Instituto Divina Misericordia. Capacitador en Investigación, Normas de Redacción Científica, Tópicos Administrativos. Asesor y jurado de tesis. Cuenta con producción científica, a razón, el libro Investigación para Universitarios (2019), así también, colaboró en la Revista Diligentia (2019). 


\section{PRODUCTION OF ACADEMIC PAPERS}

AвSTRACT: In the integral context of man, currently, it is required to demonstrate a set of general and specific competencies, which allow a comprehensive preparation of future professionals to face the expectations of the dynamic labor market. In this sense, it is necessary to develop research skills in university students in order to produce scientifically. Therefore, the present research aimed to characterize the investigative skills that higher education students must present in order to achieve scientific constructs. The methodology used was the systematic review of the scientific literature in order to determine the state of the issue, with the discussion through primary sources that approximate the subject of study, the same that will contribute to the critical and rigorous analysis. For this, academic search engines of high-impact scientific journals from the last five years were used. In summary, through the systemic level, the peculiarities of the investigative skills were determined with respect to the successful scientific production of university students.

KEYwords: Aptitude, Intellectual actívate, rifé skills, professional skills, transdisciplinary research, research program. 
Madre Celestial, Sedes Sapientiae. Para mis padres, Victor (Mi ángel del cielo) y Alicia. Mi esposa Daysi y mis hijos: Jesús, José y William.

\section{William Jesús Rojas Gutiérrez}

A Dios, al Divino Niño Jesús y a María Santísima de Yauca, por estar siempre conmigo en mi quehacer diario y colmarme de muchas bendiciones.

A mis queridos padres, Celia y Lorenzo, a mi estimada hermana Rocio por su apoyo incondicional en todo momento de mi vida y por la confianza que depositan en mi día a día. A mi pequeño y querido Benjamín, por alegrarme los dias con esa sonrisa y expresiones de afecto que hacen que cada día sea especial.

Abel Alejandro Tasayco Jala

1. Revisión conceptual de competencias y habilidades investigativas: una mirada al caso de la educación superior a nivel pregrado

7 n la educación superior se debe priorizar las habilidades investigativas para la producción de trabajos académicos, con la finalidad de incrementar la producción científica. A partir de la Ley Universitaria 
30220 en la formación universitaria, se puso de manifiesto la prioridad y necesidad de impulsar la investigación científica en la educación superior como un aspecto básico a ser desarrollado y evaluado para los procesos de acreditación exigidos desde la SUNEDU (Superintendencia Nacional de Educación Superior Universitaria) (Alarcón, Flores y Alarcón, 2018). Se plantó la necesidad de brindar calidad en la formación Universitaria, mediante la producción de investigación en los docentes y estudiantes.

Por ello, Guerrero (2007, citado en Rojas \& Aguirre, 2015) señala alrededor del término "competencia", "categorías que articulan clases de saberes, habilidades y actitudes asociados a ámbitos de desempeño específicos" (p. 205).

Hablar de la caracterización de las habilidades investigativas en la producción de trabajos académicos es presentar o describir una cosa con sus rasgos característicos del hecho fenomenológico de habilidades investigativas necesarias en los estudiantes y docente constituye una necesidad, debido a que la investigación no solo es uno de los procesos sustantivos de la Universidad, sino que representa una función específica de la labor profesional.

Machado et al. (2008) las acciones vinculadas al desarrollo de las habilidades investigativas implican:

- Modelar: observar la situación; precisar los fines de la acción; establecer dimensiones e indicadores esenciales para ejecutar la acción, anticipar acciones y resultados.

- Obtener: localizar, seleccionar; evaluar, organizar, recopilar la información.

- Procesar: analizar, organizar, identificar claves; reelaborar la información, comparar resultados. 
- Comunicar: analizar la información, seleccionar la variante de estilo comunicativo según el caso; organizar la información; elaborar la comunicación.

- Controlar: observar resultados, comparar fines y resultados; establecer conclusiones esenciales; retroalimentar sobre el proceso y los resultados de la acción (p.165).

\section{Clasificación de habilidades investigativas}

\subsection{Problematizar la realidad}

Refiere al acercamiento del investigador a la realidad, insertarse, conocer, comprender y poder analizar. Se busca que de manera inductiva nazcan las preguntas orientadoras y se formule el problema.

Para Huarancca (2020), “es hacerle preguntas inteligentes a la misma, es decir, saber distinguir y clasificar las distintas variables que la componen para desarrollar hipótesis sobre sus relaciones, dimensiones y sentidos" (p. 55).

Según Figueroa y Gómez (2015), "Se utilizan dichas experiencias como un recurso para problematizar el "ser" en su relación con situaciones prácticas con la meta de redefinir sus creencias o práctica" (p. 35).

Refiere a realizar una mirada holística de la realidad y llegar a comprender la importancia de los hechos y fenómenos sociales, creando relación dialéctica, el método es inductivo. Esto significa que la realidad estudiada es más importante que los preceptos teóricos. Si se trata de una investigación profunda y en extenso, o para una tesis, si es necesario elaborar 
un documento de Referentes teóricos y efectivamente citar a los autores originales. Esto solo servirá para finalmente contrastar los supuestos de la investigación.

\subsection{Teorizar la realidad}

En opinión de Aldas, Ávila y González (2020), "Las habilidades investigativas problematizar, teorizar y comprobar posibilitan la comprensión de la lógica del proceso investigativo" (p. 45). Cabe mencionar que la teoría en la investigación cualitativa es referencial, no determinante. El investigador genera teoría, da origen a conocimiento.

En cambio, en el enfoque cuantitativo (se trata de un marco teórico, pues posee método deductivo), la teoría de verifica, se relaciona con otras teorías.

\subsection{Comprobar la realidad}

Se refiere a la confrontación de la realidad problemática con los supuestos planteados, es la síntesis de la investigación, que proporciona o comprueba teoría. Es la etapa de evaluación y de alternativas científicas de solución a los problemas de la realidad.

En el caso de Herrera, Fernández y Horta D (2012), comprobar la realidad es la verificación permanente del proceso y los resultados de la aplicación de propuestas que constituyen alternativas científicas de solución a los problemas de la realidad, lo que permite evaluar sus logros y dificultades desde posiciones científicas y éticas, que contribuyan a su perfeccionamiento continuo a partir de su introducción en la práctica. (p. 8).

\section{Metodología}




\subsection{Enfoque}

El enfoque de la investigación será cualitativo, ya que no se tratará de medir una variable o comprobar alguna teoría. Por el contrario, se estudiará y profundizará ciertas cualidades de un fenómeno o situación. Antuna (2015), lo sustenta expresando que "esta investigación se centra en sintetizar, esquematizar, comprender un proceso, más que solo medirlo, es decir, se concentra en la profundidad y comprensión de un tema más que en la descripción o medición del mismo" (p. 33).

El enfoque cualitativo, ya que busca indagar información relevante y realizar descripciones sobre las categorías de investigación ya presentadas anteriormente, y no se hará uso de datos númericos o estadísticos.

Hernandez y Mendoza (2018) afirman sobre la investigación cualitativa:

En este tipo de enfoque también se estudian fenómenos de manera sistemática. Sin embargo, en lugar de comenzar con una teoría y luego "voltear" al mundo empírico para confirmar si esta es apoyada por los datos y resultados, el investigador comienza el proceso examinando los hechos en sí y revisado los estudios previos, ambas acciones de manera simultánea, a fin de generar una teoría que sea consistente con lo que está observando que ocurre. (p.7)

El presente proyecto está enfocado en términos de investigación al ámbito cuantitativo; puesto que, siguiendo una serie de procesos lógicos 
ayudados con instrumentos de medición como la encuesta, la cual se detallará posteriormente, se intenta demostrar una serie de datos probabilísticos.

\subsection{Nivel}

La investigación con alcance descriptivo busca especificar propiedades, características, perfiles de las personas, grupos, comunidades y procesos que se sometan a un análisis. De igual manera, pretende recoger información de manera independiente o conjunta de las variables que se investiguen. A través del alcance descriptivo se podrá realizar la descripción, caracterización e identificación de lo que acontece con el fenómeno de estudio.

\subsection{Tipo}

Hernández y Mendoza (2018) refieren que la investigación básica o pura pretende producir nuevos conocimientos y teorías con la finalidad de conseguir un conocimiento más detallado y acrecentar la base teórica existente.

La investigación básica, pura, teórica o fundamental, tiene como objetivo recopilar información de la realidad, buscando confirmar una teoría, con la finalidad de ampliar conocimientos teórico-científicos, sin tener que manejar los resultados en problemas de índole práctico, pretendiendo la absolución de interrogantes para que los conocimientos generados puedan ser aplicados en otras investigaciones.

Esto lo sustenta Baena (2017) al expresar que las investigaciones teóricas "proponen conocer las leyes generales de los fenómenos estudiados, elaborando teorías de amplio alcance para comprenderlos, y que se 
desentienden — al menos en forma inmediata — de las posibles aplicaciones prácticas que se pueda dar a los resultados" (p.17).

Cabe añadirla descripción que sobre el tipo nivel básico o puro da Esteban (2018): "Se dice que es básica porque sirve de cimiento a la investigación aplicada o tecnológica; y es fundamental porque es esencial para el desarrollo de la ciencia” (p. 1).

\subsection{Diseño: Documental}

La investigación documental es una disciplina instrumental, como cualquier actividad de tipo metodológica. Considerando desde este punto de vista, la base teórica tendría que ser sustentada por la Metodología entendida como teoría del método- o la Epistemología — Teoría o Ciencia de la Ciencia-.

Arias (2016) nos dice al respecto que: "La investigación documental es un proceso basado en la búsqueda, recuperación, análisis, critica, e interpretación de datos secundarios, es decir, los obtenidos y registrados por otros investigadores en fuentes documentales: impresas, audiovisuales o electrónicas" (p. 27).

Esto lo sustenta Fernández et al. (2019) al afirmar que las revisiones sistemáticas "son estudios secundarios que buscan responder una pregunta de investigación para lo cual realizan búsquedas exhaustivas de la evidencia disponible (estudios que hayan respondido a dicha pregunta de investigación) y sintetizan los resultados encontrados en dichas investigaciones" (p. 158).

\subsection{Técnica}


La revisión sistemática alude a un proceso de identificar sistemáticamente y evaluar varios estudios del mismo tipo y con un objetivo común. Constituye un método científico para resumir la literatura a partir de un protocolo específico que determina los estudios que se incluirán, el cual permite a otros investigadores reproducir el proceso.

Escudero y Cortez (2018) mencionan que el análisis de contenido "es un procedimiento que permite examinar textos con el propósito de conocer tanto su significado expreso o latente, así como obtener información respecto de su modo de producción" (p. 64).

La técnica que se va utilizar es el análisis de contenido. Monje (2011) señala que "el análisis de contenido es un método que busca descubrir la significación de un mensaje, ya sea este un discurso, una historia de vida, un artículo de revista, un texto escolar, un decreto ministerial, etc." (p. 157). Se trata de una técnica que nos será de utilidad, ya que deseamos elaborar una descripción sistemática del contenido para luego interpretarlo.

\subsection{Instrumento}

El instrumento a utilizar es la ficha de análisis contenido. Según Galeano (2004):

El registro de información a través de las fichas de contenido apoya el análisis e interpretación de información, en cuanto permite soportar o respaldar los hallazgos e interpretaciones; contrastar conceptos, teorías o categorías de manera sistemática, servir de base para la construcción y afinación de categorías de análisis; posibilitar la triangulación de teorías, informantes, fuentes o técnicas de recolección de información; fundamentar la construcción de la memoria metodológica; diferenciar las 
percepciones y argumentos del investigador de los informantes; evidenciar vacíos y saturaciones de informaciones de información y utilizar toda la información disponible y necesaria. (p. 50)

Las habilidades investigativas integradoras de Machado et al. (2008),

...tales como MODELAR, OBTENER, PROCESAR, COMUNICAR Y CONTROLAR; los autores sustentan su clasificación de forma piramidal donde encontramos habilidades partiendo del reconocimiento de solucionar problemas como la habilidad investigativa de mayor grado de integración; mientras que modelar, obtener, procesar, comunicar y controlar se definen como acciones de la habilidad integradora. (p. 63)

Dichas habilidades investigativas, al integrarse, genera el proceso б. de investigación ejemplificado en la Figura 1. Dichas habilidades han de presentarse como alternativas de solución en la formación universitaria de cara a la realización profesional.

\section{Figura 1}

Habilidad investigativa de mayor grado de integración 


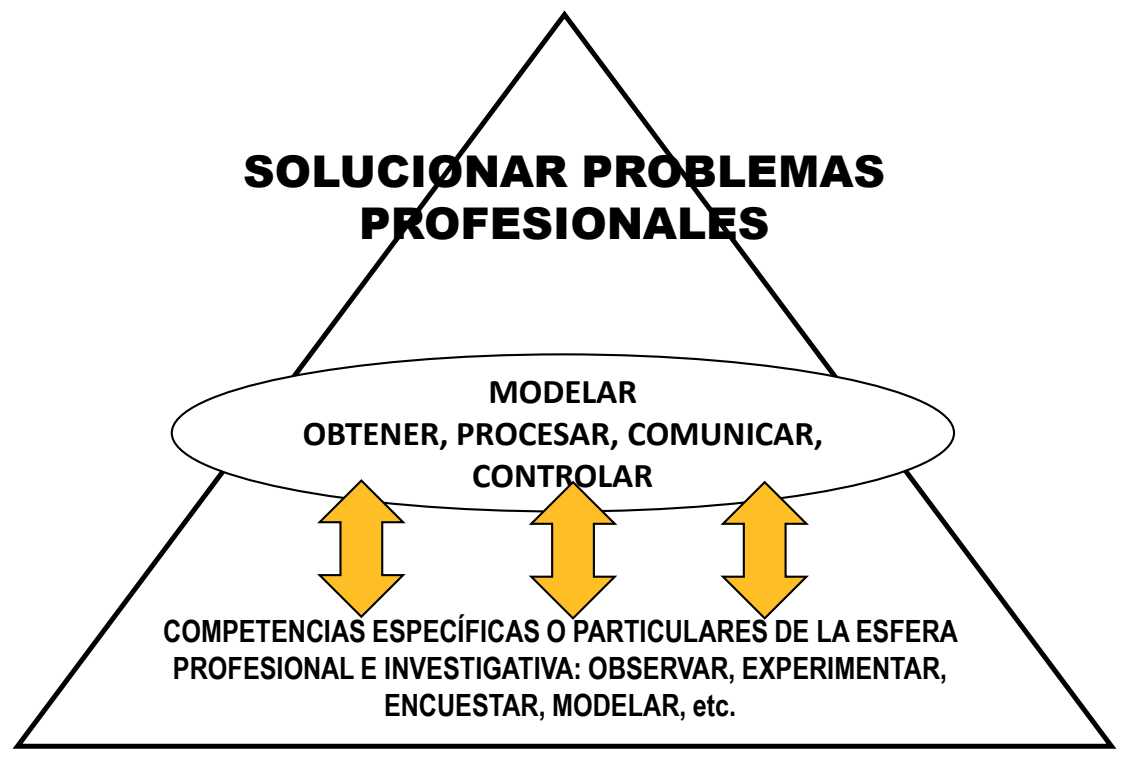

Tomado de "El desarrollo de habilidades investigativas como objetivo educativo en las condiciones de universalización de la educación superior", por Machado et al., 2008, (p.166).

Se debe considerar, a partir de lo mencionado, la importancia de la figura del mentor en investigación. Los estudiantes necesitan una persona que les motive a seguir los procesos para desarrollar habilidades investigativas.

Del mismo modo, la característica de las habilidades investigas debe superar la complejidad del proceso de solucionar problemas, convirtiéndose en oportunidad de aprendizaje. 


\section{REFERENCIAS}

Arias, F. (2016). El Proyecto de investigación: Introducción a la metodología cientifica (7. $\left.{ }^{\mathrm{a}} \mathrm{ed}.\right)$. Editorial Episteme.

Aldas, H., Ávila, C., \& González, Y. (2020). Formación de habilidades investigativas en estudiantes de Cultura Física. Revista Killkana Sociales, 4(1), 43-48. DOI: https://doi.org/10.26871/killkana_ social.v4i1.616

Antuna, P. (2015). Guía de procedimientos para la elaboración y presentación del proyecto de investigación de tesis. Universidad Juárez del Estado de Durango. https://issuu.com/carlosmartineztorres/docs/guia_de_ procedimientos_para_la_elab

Baena, G. (2014). Metodología de la investigación (3. ${ }^{\mathrm{a}}$ ed.). Grupo Editorial Patria.

Esteban, N. (2018). Tipos de investigación. Repositorio Institucional Universidad Santo Domingo de Guzmán. http://repositorio.usdg. edu.pe/bitstream/USDG/34/1/Tipos-de-Investigacion.pdf

Escudero, C. \& Cortez, L. (2018). Técnicas y métodos cualitativos para la investigación cientifica. Editorial Utmach.

Fernández, J.; Zafra, J.; Goicochea, S. \& Taype, A. (2019). Aspectos básicos sobre la lectura de revisiones sistemáticas y la interpretación de metaanálisis. Revista Acta Med Perú, 36(2), 157-169. http://www.scielo. org.pe/scielo.php?pid=S1728-59172019000200013\&script=sci_ abstract 
Concepción Obregón, T., Fernández Lorenzo, A., Matos Rodríguez, A., \& Calero Morales, S. (2017). Habilidades profesionales de intervención clínica según modos de actuación de estudiantes de tercer año de Estomatología. Educación Médica Superior, 31(1). http://www.ems.sld.cu/index.php/ems/article/view/941/478

Figueroa, I. \& Gómez, M. (2015). Cuestionar y problematizar la propia práctica: Investigación Acción Transformadora en los procesos de desarrollo profesional docente. SUMMA PSICOLÓGICA UST, 12(2), 31-42. DOI: https://doi.org/10.18774/448x.2015.12.258

Filho, F. (2015). Juegos cognitivos en el proceso decisorio. InterSienciePlace, 2(7). http://www.interscienceplace.org/isp/index.php/isp/article/ view/70

Herrera, G, Fernández, Z., \& Horta, D. (2012). Estrategia para la formación de habilidades. Investigativas en estudiantes de medicina Rev. Ciencias Médicas, 16(4), 98-112. http://scielo.sld.cu/pdf/rpr/ v16n4/rpr11412.pdf

Huarancca, E. (2020). Aplicación del método dialéctico en el desarrollo de habilidades investigativas. Editorial Área de Innovación y Desarrollo. https://www.3ciencias.com/wp-content/uploads/2020/07/ A P L I C A C I \% C 3\%93 N - D E L - M \% C 3\% 89 T O D O DIAL\%C3\% 89CTICO-EN-EL-DESARROLLO-DEHABILIDADES-INVESTIGATIVAS.pdf

Machado, E., Montes de Oca, N. \& Mena, A. (2008). El desarrollo de habilidades investigativas como objetivo educativo en las condiciones de universalización de la educación superior. Pedagogía universitaria, 13(1), 156-180. 
Machado, E. \& Montes de Oca, N. (2009). El desarrollo de habilidades investigativas en la educación superior: la solución de problemas y el eslabón gestionar información. Rev. Humanidades Médicas, 9(3). http://scielo.sld.cu/scielo.php?script=sci_ arttext\&pid=S1727-81202009000300002

Martínez, D. \& Márquez, D. (2015). Las habilidades investigativas como eje transversal de la formación para la investigación. Tendencias Pedagógicas, 24, 347-360. https://revistas.uam.es/ tendenciaspedagogicas/article/view/2110

Mendoza, L., Colunga, S., \& Fernández, P. (2018). Análisis epistemológico de la formación de habilidades sociales en educandos con Síndrome Down. Opuntia Brava, 10(4), 201-212. http://opuntiabrava.ult. edu.cu/index.php/opuntiabrava/article/view/629

Rivas, L. (2011). Las nueve competencias de un investigador. Investigación Administrativa, (108), 34-54. https://www.redalyc.org/ pdf/4560/456045339003.pdf

Rojas Salazar, A., Castro Llaja, L., Siccha Macassi, A., \& Ortega Rojas, Y. (2019). Desarrollo de habilidades investigativas en estudiantes de enfermería: Nuevos retos en el contexto formativo. Investigación Valdizana, 13(2), 107-112. http://revistas.unheval.edu.pe/index. $\mathrm{php/riv/article/view/236}$

Rojas, C. \& Aguirre, S. (2015). La formación investigativa en la educación superior en América Latina y el Caribe: una aproximación a su estado del arte. Eleuthera, 12, 197-222.

Rosell, W. \& Paneque, E. (2009). Consideraciones generales de los métodos de enseñanza y su aplicación en cada etapa del aprendizaje. Rev 
CARACTERIZACIÓN DE LAS HABILIDADES INVESTIGATIVAS EN LA PRODUCCIÓN DE TRABAJOS ACADÉMICOS

haban cienc méd, 8(2). http://scielo.sld.cu/scielo.php?script=sci_ arttext\&pid=S1729-519X2009000200016

Sampieri, R. (2010). Metodología de la investigación. Ediciones Mcgraw-hill / Interamericana Editores. 
